\title{
A team effort in Nepal: experiences from managing a large COVID-19 rehabilitation hospital outbreak
}

\author{
Raju Dhakal ${ }^{1}$. Colleen O'Connell $\mathbb{1}^{2,3} \cdot$ Jas Bahadur Gurung ${ }^{1,4}$. Ram Prakash Shah ${ }^{1} \cdot$ Hari Prasad Adhikari ${ }^{5}$. \\ Nita Chandi ${ }^{1}$ - Christine C. Groves $\mathbb{B}^{1,6}$
}

Received: 9 November 2020 / Revised: 8 December 2020 / Accepted: 20 December 2020

(c) The Author(s), under exclusive licence to International Spinal Cord Society 2021

\section{Introduction}

In August 2020, Spinal Injury Rehabilitation Centre (SIRC) experienced the largest COVID-19 outbreak among individuals with spinal cord injury (SCI) to date. We believe our experience and lessons learned may help others in the SCI community with future prevention, mitigation, and response planning.

SIRC is a free-standing, 51-bed acute rehabilitation hospital in the lower-middle income country of Nepal. Our daily census is above capacity, averaging 70 patients. We provide care for $\sim 300$ individuals with SCI and 50 individuals with stroke or traumatic brain injury every year. Average age of individuals with SCI is 36 years and stroke is 57 years. All individuals admitted are required to have a caregiver (usually an immediate family member) stay with them to assist with daily activities. Many patients have several family members staying with them. Patients stay in large wards containing 20-30 hospital beds. Caregivers sleep on low beds next to patients. Meals are taken from one communal cafeteria and are eaten in common areas.

The center is located one hour outside the capital city. Our 74 staff members commute to SIRC using both public transportation and a shared staff bus.

Raju Dhakal

dr.rajupmr@gmail.com

1 Department of Physical Medicine and Rehabilitation, Spinal Injury Rehabilitation Centre, Sanga, Nepal

2 Department of Physical Medicine and Rehabilitation, Stan Cassidy Centre for Rehabilitation, Fredericton, NB, Canada

3 Faculty of Medicine, Dalhousie University, Halifax, NS, Canada

4 Bangabandhu Sheikh Mujib Medical University, Dhaka, Bangladesh

5 Spinal Injury Rehabilitation Centre, Sanga, Nepal

6 Department of Physical Medicine and Rehabilitation, Indiana University School of Medicine, Indianapolis, IN, USA
The first COVID-19 case was diagnosed in Nepal in January 2020 [1]. At this time, our staff began work to reduce the risk of COVID-19 entry and spread within SIRC. Knowing that our setting was high risk due to open wards and crowded facilities, we have been on high alert for COVID-19 from the beginning.

\section{Pre-SIRC outbreak}

Our initial activities included the following, based on CDC and WHO guidelines for healthcare facilities:

- No-touch temperature and symptom screening of all staff, patients, and family members upon entry to SIRC.

- Limiting patient visitors to one caregiver.

- Strict masking of all staff; due to supply shortages, only clinical staff used surgical masks; all staff were given two new cloth masks (and replacements as needed) that were washed daily after use.

- Masking encouraged for all patients and family members.

- Reinforcement of strict hand hygiene of all staff, patients, and family members.

- Procurement of additional PPE: surgical masks, N-95 masks, KN-95 masks, eye protection, gowns, and gloves.

- Procurement of an additional autoclave to improve sterilization turnaround of reusable materials.

- Facility contingency planning for COVID-19 related staffing shortages; clinical staff began staying in the center for 7 days of duty, followed by 7 days off duty at home. We re-designated our recreational hall as the female dorm. Administration space was re-designated for the male dorm. All beds were spaced at least $2 \mathrm{~m}$ apart. This minimized staff need for public transportation (and therefore exposure risk), while allowing us to carry out more accurate contact tracing if needed. 
- Re-designation of administrative and vocational rehabilitation space for isolation rooms/wards and quarantine rooms/wards; controlling flow of people through the building to minimize contact.

- Education for staff, patients, and families specific to COVID-19 prevention and recognition.

- Coordination with multiple parties for management and potential referral of individuals with severe COVID-19: our designated COVID-19 referral hospital; an intensivist for potential ICU/ventilator needs within our hospital; local municipality Case Investigation and Contact Tracing; District Health Office; Ministry of Health and Population, Nepal.

Cases in Nepal began dramatically increasing in June 2020 [1]. At this time, we began seeing increased cases within the community immediately surrounding our center. We continued with the above measures and implemented additional strategies to prevent entry and spread of COVID-19:

- Telemedicine and telerehabilitation services replaced our general and rehabilitation outpatient clinic services.

- Increased pre-admission screening of patients prior to admission to SIRC. (This was limited to identifying patients with symptoms that may be related to active COVID-19 infection within 14 days of scheduled transfer and delaying transfer if indicated. Testing hospitalized patients for COVID-19 prior to SIRC admission was not possible at this time.)

- Standardized PPE use. From January to July, we acquired additional PPE that allowed for the following new protocols:

- Surgical or KN-95 mask, eye protection (face shield or goggles), head-to-toe polyurethane gown, and boots for clinicians performing new admissions.

- Surgical or KN-95 mask, eye protection, head cover, and washable gown for clinical staff providing close-contact services like therapies, wound care, dressing changes, etc.

- Surgical masks were replaced daily; KN-95 masks were replaced every 2-4 days.

- Triaging patient needs: rehabilitation was intensified for patients near-ready for discharge in order to expedite safe discharges home; new patients and/or any patient requiring intensive therapies continued to receive these therapies with appropriate PPE as above; for increasingly independent patients, caregivers were taught how to provide hands-on assistance during therapy sessions, with physically distanced supervision provided by our staff therapists; around the clock physician and nursing care was maintained.

\section{SIRC outbreak}

On August 18, 2020 a nonclinical staff member was diagnosed with COVID-19 by PCR testing, after developing fever, mild sore throat, myalgias, and abdominal pain. This prompted immediate isolation at home, followed by treatment in a designated COVID-19 hospital. We began focused contact tracing and COVID-19 PCR testing of close contacts. During this initial round of testing, 38 additional individuals were identified as COVID-19-positive, prompting isolation of these individuals and contact tracing. We converted a large conference hall and one of our therapy gyms to isolation wards. Close contacts quarantined on site. We then completed facility-wide testing for COVID-19. Individuals tested included patients, caregivers, and hospital staff (clinical and nonclinical). In total, 103/238 individuals tested COVID-19-positive (See Figs. 1 and 2).

Clearly, our immediate need was to isolate individuals with COVID-19, quarantine close contacts, and take every measure possible to protect our staff and patients. We set up a PPE donning/doffing zone and followed WHO guidines for PPE use as closely as possible. Though these supplies
Fig. 1 COVID-19 at SIRC.

Major prevention and outbreak response events by date, 2020 .

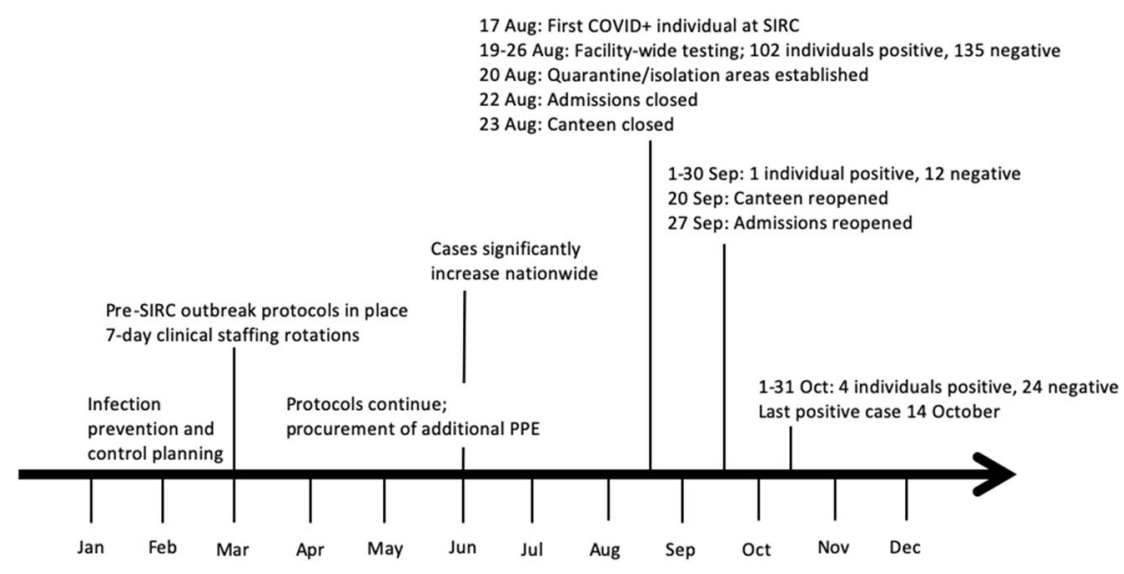


Fig. 2 COVID-19 testing at SIRC. Positive/negative PCR test results by date, August 2020 outbreak.

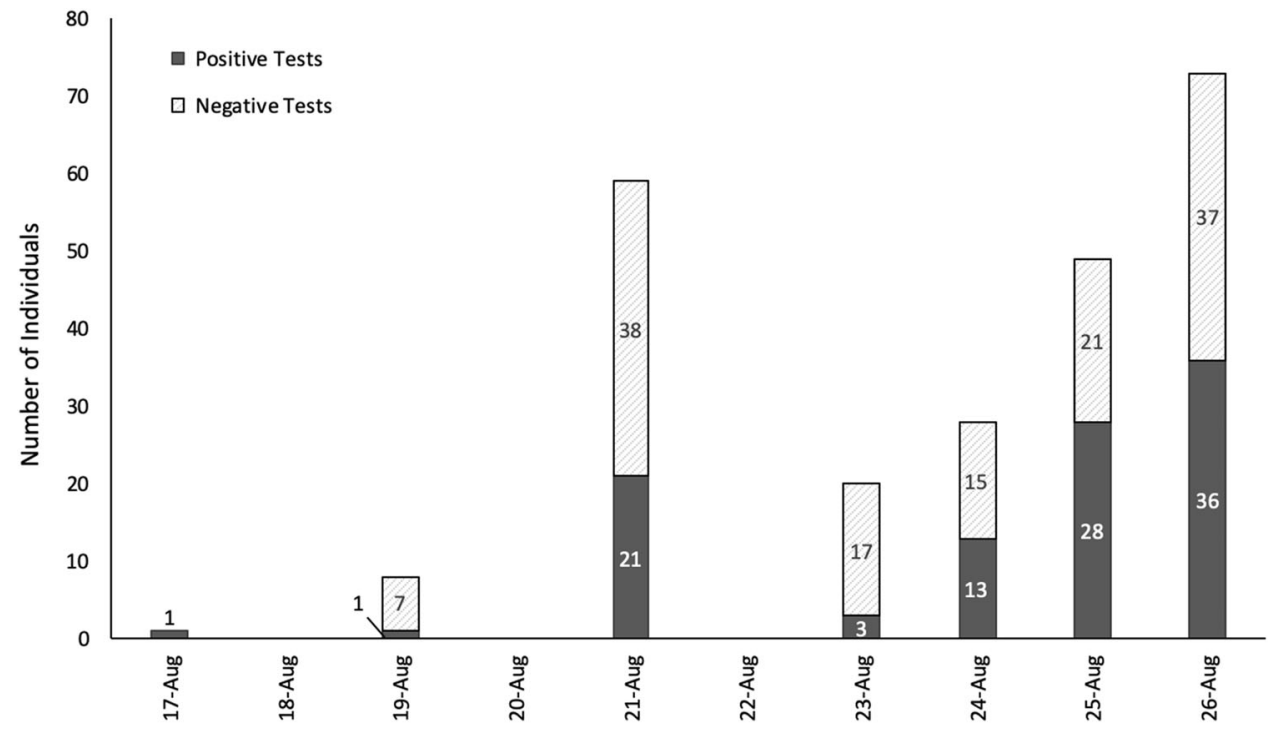

were limited, we were able to autoclave reusable items to extend our resources. In our setting, we simply don't have the space for isolation/quarantine in individual rooms. Additionally, for most of our patients with SCI, the government-established isolation hospitals were not accessible. Therefore, working with the Ministry of Health and Population we converted our rehabilitation center to a government-supported COVID-19 isolation center. Isolation wards were set up within our center for COVID-19+ individuals with SCI and their caregivers. Caregivers who tested negative for COVID-19 but whose family member was positive were given the option to self-quarantine separate from the patient. (However, in all cases, these caregivers chose to continue providing patient care with appropriate PPE.) Isolation wards were also set up for staff members and patient caregivers who tested positive for COVID-19. Pathways were established for appropriate levels of patient care. In our setting, we were able to provide medical care for individuals with mild or moderate COVID19 symptoms. Due to staffing shortages, asymptomatic COVID-19-positive staff members were allowed to provide care for COVID-19-positive patients. This was voluntary, with staff and patients both consenting to this plan. Individuals with severe symptoms were transferred to our designated COVID-19 referral hospital with ICU capability. In addition to isolation and supportive care, quarantine units were organized for close contacts who for any reason were unable to quarantine at home. This included patients, caregivers, and staff members.

Additional services were significantly altered to minimize contact among individuals within the center. Most notably, admissions were halted while the outbreak was contained. Our therapy gym was initially shut down, and individuals were treated by therapists at the bedside with appropriate PPE. Eventually, we were able to dedicate additional space within the hospital to provide more active therapy for patients, while maintaining strict physical distancing and PPE precautions. Outdoor group therapy sessions were started, which anecdotally provided significant psychological benefit to patients and staff, alike. Our cafeteria was temporarily shut down to minimize movement and exposure risk within the center, and pre-packaged meals were brought in by a catering service.

\section{Current situation}

Ultimately, 43 patients, 35 caregivers, 19 staff, and 6 vocational training participants tested positive during this outbreak, for a total of 103 individuals. Only one of the 103 individuals who tested positive required referral to a designated COVID-19 hospital for treatment.

Since the initial outbreak, we've had five sporadic COVID-19 cases (and 36 negative tests) over 2 months. Continued isolation and contact tracing with quarantine have limited spread. We have reopened admissions with all patients undergoing COVID-19 testing prior to admissions. Strict physical distancing, hand hygiene, and PPE use continue. The cafeteria has reopened with staggered mealtimes and meal delivery to patients at the bedside. We continue work to expand our telerehabilitation capacity to cover portions of routine inpatient services.

\section{Conclusion}

We've learned critical lessons that will help us and similar hospitals better prepare for the future. Having plans and protocols in place before our first hospitalized individual with COVID-19 was critical. This enabled us to adapt quickly with input from our interdisciplinary team, local/national officials, 
international experts, and patients and their family members. Strong patient and family input led to our family policy, allowing one family member to stay with patients, even if COVID-19-positive. We educated patients and their families on the risks of contracting COVID-19. 100\% of family members chose to stay with their loved one. We deeply respect their choices and believe family presence provided critical support to our patients. Government support and coordination with our designated COVID-19 referral hospital enabled us to complete testing quickly and comprehensively. Had we been more consistent and comprehensive with physical distancing requirements prior to the outbreak, we would have been able to quarantine and test more efficiently. This would have allowed for more continuity in patients' rehabilitation treatment plans and less strain on staff and facility resources. Additionally, having a separate isolation facility that is fully accessible for individuals with SCI would have reduced the risk of further spread within our center.

As we look to the future, COVID-19 cases continue to rise in Nepal. We must remain diligent and vigilant, working collaboratively to continuously improve our infection prevention strategies. This is the only way we can ensure that individuals with SCI continue to receive comprehensive rehabilitation services without interruption.

Acknowledgements We are deeply grateful to our colleagues at Spinal Injury Rehabilitation Center, the board of Spinal Injury Sangh Nepal and partners around the globe who care for individuals with SCI. Thank you for sharing your service, dedication, and expertise. Special thanks to the Vancouver-based SpiNepal group and the International Spinal Cord Society for technical and financial support.

\section{Compliance with ethical standards}

Conflict of interest The authors declare that they have no conflict of interest.

Publisher's note Springer Nature remains neutral with regard to jurisdictional claims in published maps and institutional affiliations.

\section{Reference}

1. World Health Organization Country Office for Nepal. Situation Update \#28-Coronavirus Disease 2019. 2020. https://www.who. int/nepal/news/detail/28-10-2020-who-nepal-situation-update. Accessed 5 Nov 2020. 